\title{
Complete chirp analysis of a gain-switched pulse using an interferometric two-photon absorption autocorrelation
}

\author{
Sang Hoon Chin, Young Jae Kim, Ho Seong Song, and Dug Young Kim
}

\begin{abstract}
We propose a simple but powerful scheme for the complete analysis of the frequency chirp of a gainswitched optical pulse using a fringe-resolved interferometric two-photon absorption autocorrelator. A frequency chirp imposed on the gain-switched pulse from a laser diode was retrieved from both the intensity autocorrelation trace and the envelope of the second-harmonic interference fringe pattern. To verify the accuracy of the proposed phase retrieval method, we have performed an optical pulse compression experiment by using dispersion-compensating fibers with different lengths. We have obtained close agreement by less than a $1 \%$ error between the compressed pulse widths and numerically calculated pulse widths. (C) 2006 Optical Society of America

OCIS codes: $\quad 140.2020,140.5960,320.7100$.
\end{abstract}

\section{Introduction}

Gain switching is known as the simplest method with superior durability among various optical short-pulse generation schemes (such as active or passive mode locking) since no external cavity or careful cavity alignment is typically required. ${ }^{1}$ Among various application areas, femtosecond optical pulse generation using gain-switched pulses is considered a promising technology for the measurement of ultrafast physical processes, ${ }^{2}$ optoelectronic terahertz time domain spectroscopy, ${ }^{3}$ and optoelectronic sampling. ${ }^{4}$ However, the conventional gain-switched optical pulse has a pulse width of several tens of picoseconds, and so, for femtosecond optical pulse generation, gainswitched optical pulses usually need multistage optical pulse compressions consisting of a number of optical amplifiers and specially designed optical fibers. ${ }^{5}$ To predict optical pulse profiles properly in time and frequency domains at each pulse compression stage, it is essential to know the parameters of the gain-switched pulse, such as pulse shape, pulse width, and, most of all, the frequency chirp information of the pulse for optimum pulse compression. ${ }^{6}$

The authors are with the Department of Information and Communications, Gwangju Institute of Science and Technology, 1 Oryong-dong, Buk-gu, Gwangju 500-712, South Korea. Y. J. Kim's e-mail address is youngjae@gist.ac.kr.

Received 4 April 2006; accepted 24 May 2006; posted 31 May 2006 (Doc. ID 69629).

0003-6935/06/297718-05\$15.00/0

(C) 2006 Optical Society of America
The gain-switched pulse is broadly regarded as having linear frequency chirp only, but the actual gain-switched pulse has almost linear chirp around the center of the optical pulse and nonlinear frequency chirp in the vicinity of leading or trailing edges. The presence of nonlinear frequency chirp cannot be overcome due to the variations of carrier density in the gain medium during the pulse buildup time. ${ }^{7}$ Because the nonlinear frequency chirp information is not simple enough to be fully analyzed analytically, Barry et al. ${ }^{8}$ suggested that frequencyresolved optical gating (FROG) should be a useful method to retrieve the frequency chirp of a gainswitched optical pulse. However, this method is complicated, sensitive to phase-matching conditions in a nonlinear crystal, and also requires relatively expensive components such as a monochrometer or a CCD array as well as a complicated algorithm to retrieve the frequency chirp information of a pulse. ${ }^{9}$

In this paper, we use a fringe-resolved autocorrelator based on two-photon absorption (TPA) with a large bandgap semiconductor photodiode to avoid the phase-matching problem associated with a nonlinear optical crystal ${ }^{9,10}$ and suggest a simple but accurate measurement method for the nonlinear frequency chirp of a gain-switched pulse. The pulse width and the frequency chirp information of a pulse were obtained with high precision from the intensity autocorrelation and the second-harmonic interferogram. To verify the accuracy of the retrieved frequency chirp, we perform a linear pulse compression experiment using a dispersion-compensating fiber (DCF). The measured pulse widths after propagating different lengths of a 
DCF were compared with those of numerical simulations where the optical pulse with the retrieved phase was considered as an input pulse. The linearly compressed pulse widths after propagating various lengths of a DCF agreed quite well with the numerical simulation results.

\section{Phase Retrieval Using an Interferometric Two-Photon Absorption Autocorrelator}

Figure 1 shows our experimental setup of an interferometric TPA autocorrelator. A fiber-based MachZehnder interferometer is constructed with an optical delay line in one arm of the interferometer. Various semiconductor devices, such as common photodiodes, laser diodes, light-emitting diodes, bulk semiconductors, and dielectric substrates, can be used as a two-photon absorber. ${ }^{11,12}$ In our experiment, a silicon avalanche photodiode (Si-APD) (Perkin-Elmer c30902E) with an internal multiplication factor of 100 was used. A five-phase step motor was used to scan the optical delay line rapidly and smoothly with a constant speed. The sweeping speed of the motor was $0.465 \mathrm{~mm} / \mathrm{s}$, which caused the interference fringe to occur at a frequency of $f_{d}=300 \mathrm{~Hz}$. To launch the output pulse into the Si-APD, the gain-switched light was focused circularly on the front facet of the Si-APD with approximately a $50 \mu \mathrm{m}$ diameter FWHM spot size.

The output current of the Si-APD receiver for the collinear TPA autocorrelation measurement system shown in Fig. 1 can be written as ${ }^{13}$

$$
\begin{aligned}
S(\tau) \propto & 1+2 G_{2}(\tau)+4 \operatorname{Re}\left[F_{1}(\tau) \exp \left(-i \omega_{o} \tau\right)\right] \\
& +\operatorname{Re}\left[F_{2}(\tau) \exp \left(-i 2 \omega_{o} \tau\right)\right],
\end{aligned}
$$

where

$$
G_{2}(\tau)=\int_{-\infty}^{\infty} I(t) I(t-\tau) \mathrm{d} t .
$$

$\tau$ is the delay time of the interferometer, and $\omega_{0}$ is the carrier frequency of the input pulse. $G_{2}(\tau), F_{1}(\tau)$, and $F_{2}(\tau)$ are, respectively, the intensity autocorrelation and the interferogram of the TPA light, which can be expressed as

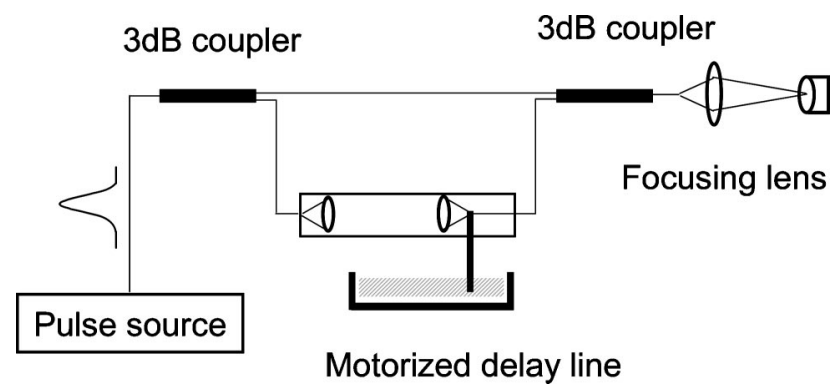

Fig. 1. Experimental setup for the fringe-resolved TPA autocorrelation measurement.

$$
\begin{aligned}
F_{1}(\tau)= & \frac{1}{2} \int_{-\infty}^{\infty}[I(t)+I(t-\tau)] \sqrt{I(t) I(t-\tau)} \\
& \times \exp \left\{i\left[\omega_{o} \tau+\varphi(t)-\varphi(t-\tau)\right]\right\} \mathrm{d} t, \\
F_{2}(\tau)= & \int_{-\infty}^{\infty} I(t) I(t-\tau) \\
& \times \exp \left\{i 2\left[\omega_{o} \tau+\varphi(t)-\varphi(t-\tau)\right]\right\} \mathrm{d} t .
\end{aligned}
$$

$I(t)$ and $\varphi(t)$ represent the intensity and the phase of a pulse, respectively. Here, the electric field of the pulse is represented as

$$
E(t)=\sqrt{I(t)} \exp \left\{i\left[\varphi(t)-\omega_{o} \tau\right]\right\}+\text { c.c. }
$$

$G_{2}(\tau)$ and $F_{2}(\tau)$ are slowly varying envelope functions with respect to the relative delay time $\tau$, which can be exploited for a detailed pulse analysis. The pulse width information of the input pulse is included in $G_{2}(\tau)$, while its phase chirp information is in $F_{2}(\tau)$. To separate $G_{2}(\tau)$ and $F_{2}(\tau)$ from an autocorrelation signal, a frequency filtering technique was employed based on their carrier-frequency difference. The $G_{2}(\tau)$ has a dc carrier, while $F_{2}(\tau)$ has a carrier frequency of $2 \omega_{o}$. To select the $G_{2}(\tau)$ signal, the whole autocorrelation trace was filtered by a low-pass filter, and for the $F_{2}(\tau)$ signal it was sent through a bandpass filter whose center frequency was at $2 \omega_{0}$. These digital filtering processes were dealt with by LabVIEW programming.

\section{Experimental Results and Discussion}

A multiple-quantum-well distributed-feedback (DFB) laser diode operating at a center wavelength of 1.55 $\mu \mathrm{m}$ was used and was characterized in our experiment. The DFB laser was biased with a $26 \mathrm{~mA} \mathrm{dc}$ current and modulated by a $28 \mathrm{dBm}$ sinusoidal signal at a $2 \mathrm{GHz}$ modulation frequency from an amplified low-noise signal source. Figure 2(a) shows the original interferometric autocorrelation signal $S(\tau)$, which includes information for both $G_{2}(\tau)$ and $F_{2}(\tau)$. Figure 2(b) shows the $G_{2}(\tau)$ plotted on a solid curve and the envelope of $F_{2}(\tau)$ on the dotted curve, which are obtained by the numerical filtering process explained above.

To reflect the asymmetric pulse shape through gain switching of the semiconductor laser in advance, we have assumed nonsymmetric Gaussian pulse intensity for a gain-switched pulse such as

$$
\begin{aligned}
I(t) & =I_{0} \exp \left[-\left(\frac{t}{T_{1}}\right)^{2}\right]: \quad t<0, \\
& =I_{0} \exp \left[-\left(\frac{t}{T_{2}}\right)^{2}\right]: \quad t \geq 0,
\end{aligned}
$$

where $I_{0}$ is the peak pulse intensity and $T_{1}$ and $T_{2}$ are constants that determine the pulse width and its 


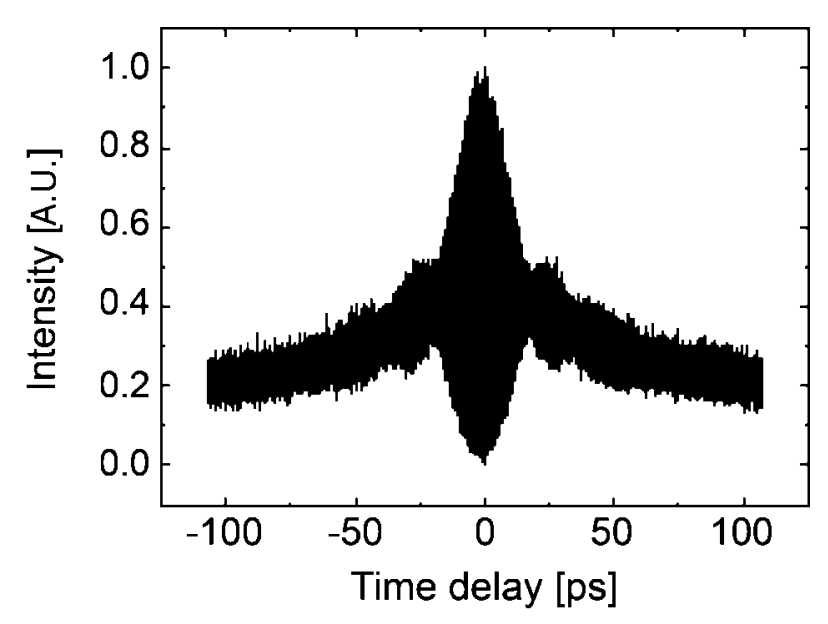

(a)

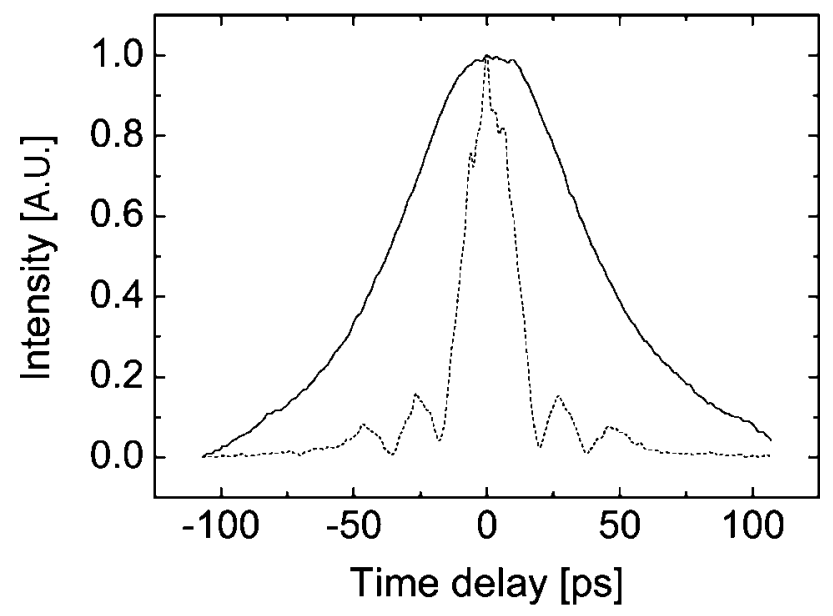

(b)

Fig. 2. (a) Interferometric autocorrelation trace $S(\tau)$ obtained directly from the output of the APD receiver. (b) Intensity autocorrelation curve $G_{2}(\tau)$ as the solid curve and the envelope for the interference fringe of $F_{2}(\tau)$ as the dotted curve.

asymmetric ratio. By fitting the $G_{2}(\tau)$ trace in Fig. 2(b) with a standard least-squares curve-fitting method, we have obtained $T_{1}=33.8 \mathrm{ps}$ and $T_{2}=$ $36.2 \mathrm{ps}$. The FWHM of the gain-switched pulse was estimated to be $58 \mathrm{ps}$ from the intensity autocorrelation trace $G_{2}(\tau)$, and the FWHM of the envelope of the $F_{2}(\tau)$ fringe signal was estimated to be approximately $20 \mathrm{ps}$, assuming a Gaussian waveform. The ratio of these two widths is 2.9 , which indicates that the gainswitched pulse has nonzero phase chirp; this ratio becomes 1 for a chirp-free optical pulse. ${ }^{12}$

It is notable that we have small wiggles in the side wings of the $F_{2}(\tau)$ trace shown in Fig. 2(b), whose form just resembles that of the nonlinear frequency chirp caused by the self-phase modulation (SPM) effect: a narrow peak at the center and small wiggles in the side wings of the trace. ${ }^{14}$ Even though the measured $F_{2}(\tau)$ trace shows similar features observed in pulses chirped by the SPM effect, the physical origin of this trace in a gain-switched pulse is quite different from the SPM effect. ${ }^{15}$ A dynamic wavelength shift of a
DFB laser under deep carrier modulation or frequency chirp in a gain-switched DFB laser pulse is caused by the carrier-induced refractive index change. In the case of a gain-switched pulse from a laser diode, the change of a laser frequency can be examined to be proportional to the integration of the pulse intensity with respect to time ${ }^{16}$ as the change in carrier density is proportional to the photon number generated in a laser cavity or the integration of the output pulse intensity from a laser cavity. This is the reason why we assumed the frequency chirp of a gain-switched pulse is a linear chirp incorporated with a nonlinear chirp, whose form is just like the effect caused by SPM. ${ }^{8}$ Therefore the phase of a gainswitched pulse can be expressed as

$$
\begin{aligned}
\varphi(t) & =-\left(\frac{C}{2}\right)\left(\frac{t}{T_{1}}\right)^{2}-S \exp \left[\left(\frac{t}{T_{1}}\right)^{2}\right]: \quad t<0 \\
& =-\left(\frac{C}{2}\right)\left(\frac{t}{T_{2}}\right)^{2}-S \exp \left[\left(\frac{t}{T_{2}}\right)^{2}\right]: \quad t \geq 0
\end{aligned}
$$

where $C$ is a linear chirping parameter and $S$ is a nonlinear chirping parameter. The instantaneous frequency $\delta \nu(t)$ across a pulse is obtained from the phase $\varphi(t)$ by $\delta v(t)=-(2 \pi)^{-1} \mathrm{~d} \varphi(t) / \mathrm{d} t$. For a complete analysis of a gain-switched pulse from the simple numerical model of Eq. (7), which is based on the carrier-induced refractive index change inside a laser cavity, the initial electric field was finally given as

$$
\begin{aligned}
E(t)= & E_{0} \exp \left[-\left(\frac{t}{T_{1}}\right)^{2}\right] \exp \left(-\frac{i}{2}\left\{\omega_{0} t+\left(\frac{C}{2}\right)\left(\frac{t}{T_{1}}\right)^{2}\right.\right. \\
& \left.\left.+S \exp \left[\left(\frac{t}{T_{1}}\right)^{2}\right]\right\}\right): \quad t<0 \\
= & E_{0} \exp \left[-\left(\frac{t}{T_{2}}\right)^{2}\right] \exp \left(-\frac{i}{2}\left\{\omega_{0} t+\left(\frac{C}{2}\right)\left(\frac{t}{T_{2}}\right)^{2}\right.\right. \\
& \left.\left.+S \exp \left[\left(\frac{t}{T_{2}}\right)^{2}\right]\right\}\right): \quad t \geq 0 .
\end{aligned}
$$

We have found optimum values for the $C$ and $S$ parameters by fitting the measured trace of $F_{2}(\tau)$ and $G_{2}(\tau)$ using a standard least-squares fitting method. Close agreement between measured $G_{2}(\tau)$ and calculated $G_{2}(\tau)$ is clearly shown in Fig. 3(a). In Fig. 3(b), a comparison was made for $F_{2}(\tau)$. The retrieved FWHM pulse width $G_{2}(\tau)$ and chirp width $F_{2}(\tau)$ were 58 and $14.6 \mathrm{ps}$, respectively.

To verify the retrieved nonlinear phase chirp obtained by our newly proposed method, we performed a linear pulse compression with a DCF and compared the actual pulse widths with expected pulse widths based on a numerical model shown in Eq. (8). If the phase of a gain-switched pulse was correctly retrieved, the compressed pulse width should match the estimated pulse width by using 


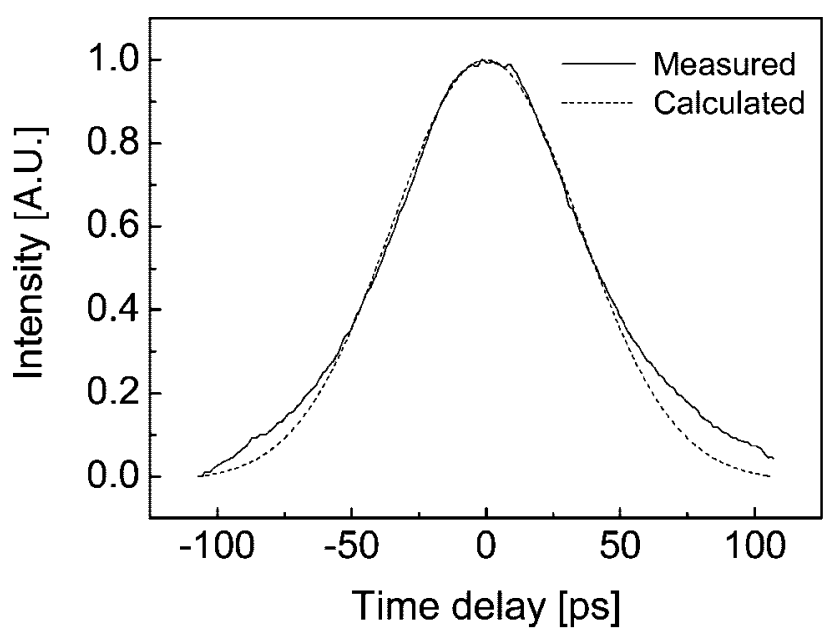

(a)

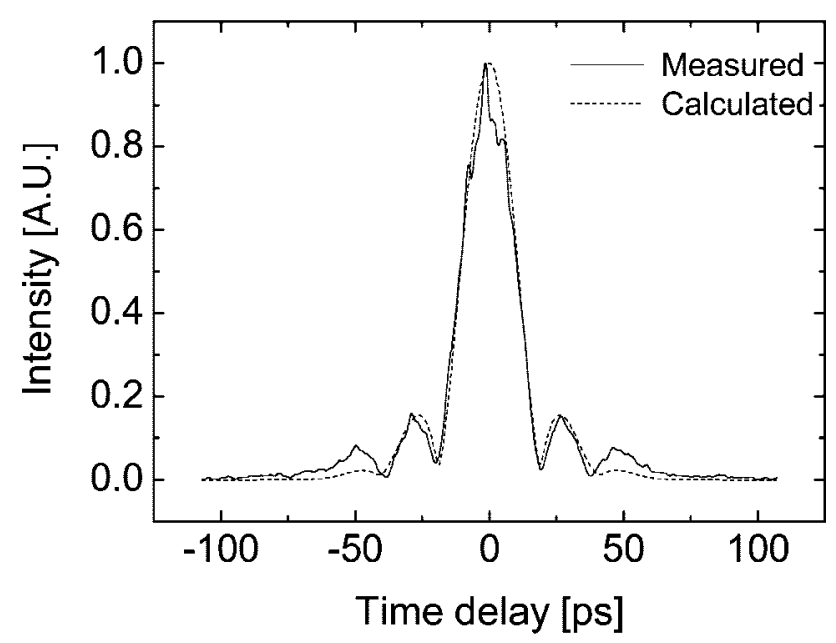

(b)

Fig. 3. Measured and calculated traces for the $G_{2}(\tau)$ and $F_{2}(\tau)$. (a) Measured $G_{2}(\tau)$ as the solid curve and the calculated $G_{2}(\tau)$ as the dotted curve. (b) Measured $F_{2}(\tau)$ as the solid curve and the calculated $F_{2}(\tau)$ as the dotted curve.

the conventional pulse-propagation equation. The nonlinear pulse-propagation effect was ignored in this pulse-propagation simulation because the peak power of the pulse is not so high for a gain-switched pulse by a laser diode. ${ }^{17}$ Figure 4 shows the retrieved pulse intensity and the corresponding frequency chirp of the gain-switched pulse just before it is injected into a DCF. The pulse intensity profile, which is identical to $G_{2}(\tau)$ in Fig. 3(a), is shown as a solid curve, and it has an asymmetric Gaussian shape. The dotted curve shows the retrieved frequency chirp of the pulse when it is assumed to be only linear, and the dashed curve is obtained under the consideration of the nonlinear frequency chirp. Output pulse widths were measured and plotted in Fig. 5 after propagating through seven different lengths of a DCF, e.g., 0.4, 1.0, 1.4, 1.9, 2.6, 3.0, and $3.6 \mathrm{~km}$. The chromatic dispersion coefficient $D$ for the $\mathrm{DCF}$ is $-65 \mathrm{ps} /(\mathrm{km} / \mathrm{nm})$. Filled circles are measured pulse widths at each length of the DCF, and they are

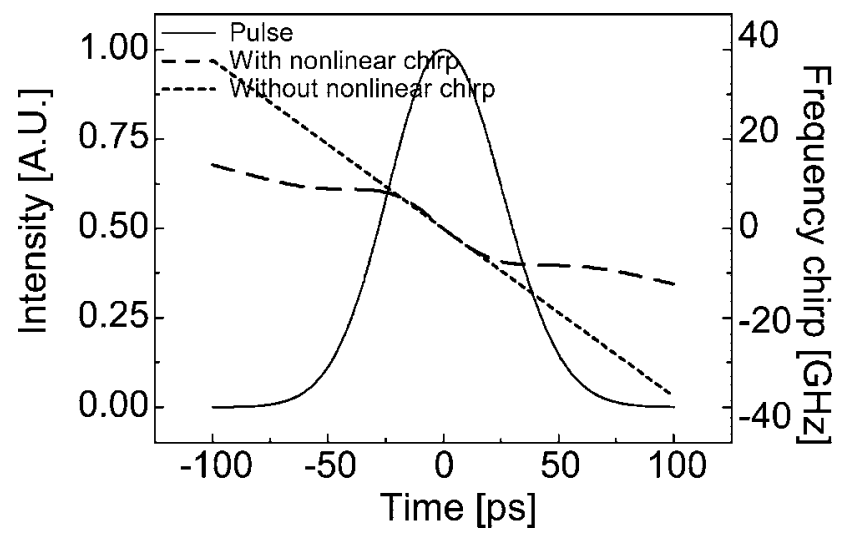

Fig. 4. Retrieved frequency chirp and intensity profiles. Pulse intensity profile, which is identical to the retrieved $G_{2}(\tau)$ in Fig. 3(a), shown as the solid curve. The dotted line describes the retrieved frequency chirp with only the linear frequency chirping term, and the dashed curve is under the consideration of a nonlinear frequency chirping term as well.

virtually connected with a curve for comparison. The dashed curve shows the expected pulse widths as a function of propagation length, which is obtained from a simple numerical analysis when only a linear frequency chirp is considered. The solid curve describes the expected pulse width when the nonlinear frequency chirp is considered. The decreasing tendency of the calculated pulse width as a function of propagation distance agrees well with the measured results with less than a $1 \%$ error when we take the nonlinear phase chirp described in Eq. (8) as the initial phase chirp of the gain-switched pulse. It shows that the minimum pulse width is $21.7 \mathrm{ps}$ after propagating the $2.6 \mathrm{~km}$ length of the DCF. When we only assume a linear phase chirp, the calculated pulse widths do not match with the experimental

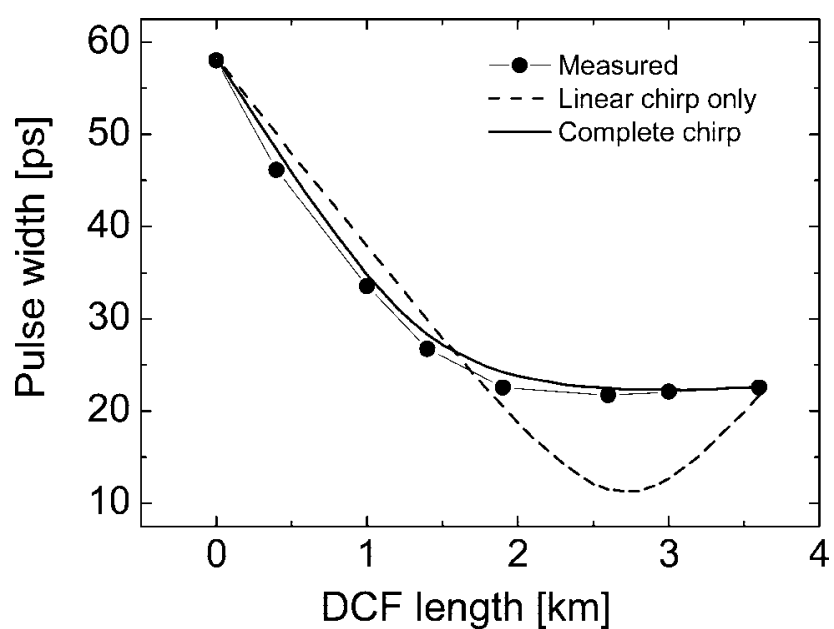

Fig. 5. Measured and calculated pulse widths after propagating different lengths of a DCF. The filled circles connected with a solid curve represent the measured pulse widths at each end of the DCF. The dashed curve represents the numerical results with only a linear chirping term, and the solid curve describes the numerically expected pulse widths including the effect of the nonlinear chirping term as well. 
pulse widths. For a pulse with just a linear frequency chirp, the pulse width is given as a parabolic function with respect to the point of the minimum pulse width. Furthermore, there is a large discrepancy between the measured minimum pulse width of $21.7 \mathrm{ps}$ and the expected minimum pulse width of $11.2 \mathrm{ps}$. Another finding through pulse compression experiment is that there is a large tolerance of the DCF length for obtaining maximum pulse compression when a gainswitched semiconductor laser is used as a seeding pulse source. When only the linear frequency chirp is included for the estimation of pulse compression, minimum pulse width can be realized within a relatively short and restricted length of a DCF. In our experiment, the pulse width reduction as a function of propagation length had an L-shaped curve because of the nonlinear frequency chirp across the pulse.

\section{Conclusion}

In this paper, we introduced a simple but powerful method of obtaining highly accurate nonlinear phase chirp of a gain-switched pulse laser. Using this scheme, we can obtain the asymmetric pulse shape and the amount of nonlinear frequency chirp across a gain-switched pulse by measuring a simple fringeresolved autocorrelation trace for a pulse. Our interferometric TPA autocorrelator is quite simple, yet the pulse characterization result is both accurate and reassuring. We believe that this method is efficient for analyzing both a gain-switched pulse and a pulse with a SPM-type nonlinear phase chirp.

This work was supported by the Ministry of Science and Technology of Korea through Creative Research Initiatives.

\section{References}

1. M. Schell, D. Huhse, and D. Bimberg, "Generation of 2.5-ps light pulses with $15-\mathrm{nm}$ wavelength tunability at $1.3 \mu \mathrm{m}$ by a self-seeded gain-switched semiconductor laser," IEEE Photon. Technol. Lett. 5, 1267-1269 (1993).

2. D. H. Auston and M. C. Nuss, "Electrooptic generation and detection of femtosecond electrical transients," IEEE J. Quantum Electron. 24, 184-197 (1988).
3. B. B. Hu and M. C. Nuss, "Imaging with terahertz waves," Opt. Lett. 20, 1716-1718 (1995)

4. J. Valdmanis and G. Mourou, "Subpicosecond electrooptic sampling: principles and applications," IEEE J. Quantum Electron. QE-22, 69-78 (1986).

5. I. Y. Khrushchev, I. H. White, and R. V. Penty, "High-quality laser diode pulse compression in dispersion-imbalanced loop mirror," Electron. Lett. 34, 1009-1010 (1998).

6. L. P. Barry, B. C. Thomas, J. M. Dudely, and J. D. Harvey, "Optimised design of fibre-based pulse compressor for gainswitched DFB laser pulses at $1.5 \mu \mathrm{m}$," Electron. Lett. 35, 1166-1168 (1999).

7. H.-F. Liu, Y. Ogawa, and S. Oshiba, "Generation of an extremely short single mode pulse ( $\sim 2 \mathrm{ps})$ by fiber compression of a gain-switched pulse from a $1.3 \mu \mathrm{m}$ distributed-feedback laser diode," Appl. Phys. Lett. 59, 1284-1286 (1991).

8. L. P. Barry, B. C. Thomsen, J. M. Dudley, and J. D. Harvey, "Characterization of $1.55-\mu \mathrm{m}$ pulses from a self-seeded gainswitched Fabry-Perot laser diode using frequency-resolved optical gating," IEEE Photon. Technol. Lett. 10, 935-937 (1998).

9. T. Sun, K. K. Bosco, I. K. Fung, G. K. Sou, L. Wong, and G. Lanzani, "Two-photon absorption autocorrelation of visible to ultraviolet femtosecond laser pulses using ZnS-based photodetectors," IEEE Photon. Technol. Lett. 14, 86-88 (2002).

10. F. R. Laughton, J. H. Marsh, D. A. Barrow, and E. L. Portnoi, "The two-photon absorption semiconductor waveguide autocorrelator," IEEE J. Quantum Electron. 10, 838-845 (1994).

11. K. Kikuchi, "Optical sampling system at $1.5 \mu \mathrm{m}$ using two photon absorption in Si avalanche photodiode," Electron. Lett. 34, 1354-1355 (1998).

12. A. M. Streltsov, K. D. Moll, and A. L. Gaeta, "Pulse autocorrelation measurements based on two- and three-photon conductivity in a GaN photodiode," Appl. Phys. Lett. 75, 3778-3780 (1999).

13. K. Mogi, K. Naganuma, and H. Yamada, "A novel real-time chirp measurement method for ultrashort optical pulses," Jpn. J. Appl. Phys., Part 1 27, 2078-2081 (1988).

14. R. Trebino, C. C. Hayden, A. M. Johnson, and A. M. Levine, "Chirp and self-phase modulation in induced-grating autocorrelation measurements of ultrashort pulses," Opt. Lett. 15, 1079-1081 (1990).

15. G. P. Agrawal and N. K. Dutta, Semiconductor Lasers, 2nd ed. (Van Nostrand-Reinhold, 1993).

16. C. Henry, "Theory of the linewidth of semiconductor lasers," IEEE J. Quantum Electron. 18, 259-264 (1982).

17. G. P. Agrawal, Fiber-Optic Communication Systems, 2nd ed. (Wiley, 1997). 